\title{
Topic maps constraint languages: understanding and comparing
}

\section{G.R. Librelotto* and R.P. Azevedo}

Universidade Federal de Santa Maria (UFSM),

Departamento de Eletrônica e Computação (DELC),

GMob - Grupo de Sistemas de Computação Móvel, Av. Roraima, 1000, Bairro Camobi, 97105-900, Santa Maria - RS, Brasil

E-mail: librelotto@inf.ufsm.br E-mail: rpa.renato@gmail.com

*Corresponding author

\section{J.C. Ramalho and P.R. Henriques}

Department of Informatics,

University of Minho,

Campus of Gualtar, 4710-057 Braga, Portugal

E-mail: jcr@di.uminho.pt_ E-mail:prh@di.uminho.pt

\begin{abstract}
Topic map constraint language (TMCL) provides a means to express constraints on topic maps conforming to ISO/IEC 13250. In this article, we will use a test suite and show, step-by-step, the way we handled several kinds of topic maps constraints in many different instances in order to answer questions like: Do they do the same job? Are there some kinds of topic maps constraints that are easier to specify with one of them? Do you need different background to use the tools? Is it possible to use them in similar situations (the same topic maps instances)? May we use them to produce an equal result? How do AsTMa!, OSL, Toma and XTche relate to TMCL? What kind of constraints each one of these three cannot specify? We will conclude this paper with a summary of the comparisons accomplished between those topic maps constraint languages over the use case proposed.
\end{abstract}

Keywords: knowledge representation; topic maps; constraint languages; XML; intelligent systems.

Reference to this paper should be made as follows: Librelotto, G.R., Azevedo, R.P., Ramalho, J.C. and Henriques, P.R. (2009) 'Topic maps constraint languages: understanding and comparing', Int. J. Reasoning-based Intelligent Systems, Vol. 1, Nos. 3/4, pp.173-181.

Biographical notes: Giovani Rubert Librelotto received his $\mathrm{PhD}$ in Computer Science at the University of Minho in 2005, in Portugal. In February 2009 he was named a Professor in the Electronic and Computer Science Department at the Federal University of Santa Maria (UFSM), RS, Brazil. He has been involved in research around processing structured documents, bioinformatics, XML and topic maps.

Renato Preigschadt de Azevedo received his Degree in Computer Science from UNIFRA, Brazil. Currently, he is an MSc candidate in Computer Science at UFSM, Brazil. He is a Computer Science Professor at URI, Brazil.

José Carlos Ramalho is a Teacher at the Department of Informatics at the University of Minho and a Researcher at the CCTC Research Center. He has a Masters on 'Compiler Construction' and a $\mathrm{PhD}$ on the subject 'Structured Document Processing and Semantics'. He is supervising several XML/SGML projects and acting as an External Consultant for several institutions. He also has been the Chair and Chief Editor of the Portuguese XML conference.

Pedro Rangel Henriques is a Teacher at the Department of Informatics at the University of Minho and a Researcher at the CCTC Research Center. He is supervising several XML/SGML projects. $\mathrm{He}$ also has been the Chair and Chief Editor of the Portuguese XML conference. 


\section{Introduction}

Semantic Web is concerned with the arrangement on the web of information in such way that its meaning can be understood by computers as easily as by people; that is, the web pages contain not only the concrete information to be shown, but also metadata that allows for its semantic interpretation. Such an organisation of information offers new perspectives for the web (Park and Hunting, 2003):

- greater efficiency and precision in the search for and comprehension of information by users, humans or machines

- automatic treatment of information

- transfer of simple tasks like search, selection, updating and transaction from the user to the system.

Organisation, standardisation and automatic treatment of information are the key elements that allowed the transition from the first web generation, which is first of all a vast collection of anarchic information, to the Semantic Web, which aims at treating decentralised, sharable and exploitable knowledge.

The Semantic Web requires the cooperation of various disciplines: ontologies, artificial intelligence, agents, formal logic, languages, graph theory and topology, etc. Our working area is ontologies for the web, more exactly, ontologies represented by topic maps to be handled by web applications and browsers.

Ontology is a way of describing a shared common understanding, about the kind of objects and relationships which are being talked about, so that communication can happen between people and application systems (Rath, 2003). In other words, it is the terminology of a domain (it defines the universe of discourse).

As a real example consider the thesaurus used to search in a set of similar, but independent, websites. Ontologies can be used to:

- $\quad$ create a structured core vocabulary, to be used and validated by a set of actors in a community

- define and use logical relationships and rules between the concepts, allowing an efficient use of intelligent agents

- develop, maintain and publish knowledge (that changes rapidly) about an organisation (the whole or a part), easily providing different views.

Topic maps (Park and Hunting, 2003) are a good solution to organise concepts, and the relationships between those concepts, because they follow a standard notation ISO/IEC 13250 (Biezunsky et al., 1999) - for interchangeable knowledge representation. Topic maps are composed of topics and associations giving rise to structured semantic network that gathers information concerned with certain domain. This hierarchical topic network can represent the ontology. A topic map is an organised set of topics (formal representation of subjects), with:
- $\quad$ several names for each topic (or subject of the index)

- $\quad$ pointers (occurrences) between topics and external documents (information resources) that are indexed

- $\quad$ semantic relationships, whether they are hierarchical or not, between topics via associations.

It also has the capability of supporting multi-classification (a topic can belong to more than one class) and offers a filtering mechanism based on the concept of scope that is associated with names, occurrences and associations.

According to Rath (2003), topic maps are very well suited to represent ontologies. Ontologies play a key role in many real-world knowledge representation applications, and namely the development of Semantic Web. The ability of topic maps to link resources anywhere and to organise these resources according to a single ontology, will make topic maps a key component of the new generation of web-aware knowledge management solutions.

On one hand, this section helps to understand our interest on topic maps in the actually important area of Semantic Web; on the other hand, the concepts so far introduced pointed out the indubitable need for mechanisms to guarantee the semantic correctness of topic maps.

Besides the simplicity and powerfulness of the topic/association-based model, there are two topic maps features that are important in the process of understanding and reasoning about a domain: the hierarchical structure that is represented in a map (defined by the relations is-a or contains) and the complementary topic network (made up of other links that connect topics that are not included in each other but just interact).

The facts above explain the importance of topic maps to describe knowledge in general; in particular their application to define ontologies is one of the growing up fields. So topic maps are nowadays widely used within XML environments: in archives, for cataloguing purposes; or in web browsers, for conceptual navigation.

To build reliable systems, like those referred, it is crucial to be sure about the validation of the underlying semantic network. When developing real topic maps, it is highly convenient to use a system to validate them; this is, to verify the correctness of an actual instance against the formal specification of the respective family of topic maps (according to the intention of its creator).

Currently, we can find three approaches to constrain topic maps - OSL (Garshol, 2004), AsTMa! (Barta, 2003), Toma (Pinchuk, 2007) and XTche (Librelotto et al., 2005) - that allow us to specify constraints and to validate the instances of a family of topic maps against that set of rules. With these resemblances it is easy to conclude that they are quite similar. However they differ in some fundamental concepts. These three topic maps constraint specification languages were thoroughly tested and benchmarked with a huge test suite. The most significant results will be discussed in this paper.

The paper is organised as follows. In the next section, the topic maps constraint languages (TMCL) are introduced. 
The used case study - an e-commerce corporation - is introduced in Section 3. Section 4 presents the comparison among the main constraint languages. Finally, conclusions are given in Section 5.

\section{TMCL}

Topic maps are an ISO standard for the representation and interchange of knowledge, with an emphasis on the findability of information. A topic map can represent information using topics (representing any concept), associations (which represent the relationships between them), and occurrences (relationships between topics and information resources relevant to them). They are thus similar to semantic networks and both concept and mind maps in many respects.

According to topic map data model (TMDM) (Garshol and Moore, 2005), topic maps are abstract structures that can encode knowledge and connect this encoded knowledge to relevant information resources. On one hand, this makes topic maps a convenient model for knowledge representation; but on the other hand, this can also put in risk the topic map consistency. A set of semantic constraints must be imposed to the topic map in order to grant its consistency.

In a general way, given a specification, a constraint is a logical expression that restricts the possible values that a variable in that specification can take.

Constraints can be applied to specifications in all domains. The set of valid sentences of a formal language can be restricted using contextual conditions over the grammar attributes. The proof process in logic programming can also be controlled adding constraints to the predicates. Also, annotated documents can be coerced completing their type definition (DTDs or XML-Schema) with constraints; for this purpose there are some domain specific languages, like Schematron (Dodds, 2001) and XCSL (Jacinto et al., 2003).

A domain specific language enables the description of constraints required by each problem in a direct, clear and simple way; moreover it allows the derivation of a program to automatise the validation task. The derived semantic validator will verify every document, keeping silent when the constraints are satisfied and reporting errors properly whenever the contextual conditions are broken.

This proposed topic maps constraint system behaves like Schematron and XCSL. It means that the processor (generated according to a specification) checks the semantic validity of a topic map: if it is correct, the result is empty; on the other hand, every error detected is reported displaying an error message.

The language to define topic map constraints is called as TMCL. This language is currently on its way for standardisation (ISO 19756) (Nishikawa et al., 2004). The objective of TMCL is to allow formal specification of rules for topic map documents. TMCL has a similar purpose as schema languages for relational databases or XML applications. The constraint language is required to formalise application of specific rules. Currently there are different proposed constraint languages that will be presented in the next subsections.

\section{$2.1 \quad X$ Tche language}

$\mathrm{XTche}$ is a process for specifying constraints on topic maps with a constraint language. This language allows to express contextual conditions on classes of topic maps. With XTche, a topic map designer defines a set of restrictions that enables to verify if a particular topic map is semantically valid.

XTche is an XML Schema oriented language (Duckett et al., 2001). This fact brings two benefits: on one hand it allows for the syntactic specification of topic maps (not only the constraints) and on the other hand it enables the use of an XML Schema editor [for instance, XMLSpy (http://www.altova.com)] to provide a graphical interface and the basic syntactic checker.

The constraining process is composed of a language and a processor (Librelotto et al., 2005). The language is based on XML Schema syntax. The processor is developed in XSLT language. The XTche processor takes a XTche specification and it generates a particular XSLT stylesheet. This stylesheet can validate a specific topic map (or a set of them) according to the constraints in the XTche specification.

As the manual checking of large topic maps (frequent in real cases) is impossible, it is mandatory to provide an automatic validator.

XTche language meets all the TMCL requirements (Nishikawa et al., 2004); for that purpose, XTche has a set of constructors to describe constraints in topic maps. But the novelty of the proposal is that the language also permits the definition of the topic map structure in an XML Schema style. An XTche specification merges the schema (defining the structure and the basic semantics) with constraints (describing the contextual semantics) for all the topic maps in that family.

\subsection{AsTMa! language}

AsTMa! (Barta, 2003) is another language for constraint topic maps aiming to validate topic maps against a given set of rules. AsTMa! is a member of AsTMa* family (which includes $\mathrm{AsTMa}=$ for authoring $\mathrm{TM}$ and AsTMa? for querying TM) and exposes some features of TMCL, because it has written earlier than the final version of the TMCL.

Resembling XTche the constraining process is composed of a language and a processor. The language is based on the Perl language and the processor is written in Perl. At this time the AsTMa! language is no longer maintained, because the author is working on a completely new distribution. So for this article we assume the AsTMa! language definition for expression evaluation. 


\subsection{Ontopia schema language (OSL)}

According to the OSL specification (Garshol, 2004), OSL has been designed to have a minimal number of features available on TMCL and a minimum expressive power.

Basically the OSL constraints only the structure of a topic map. An OSL schema consists of a set of topic and association class definitions. These class definitions constrain the structure of the instances of the classes, and so control the form information may take in a topic map that uses the schema (Garshol, 2004).

As the other two languages OSL is composed by a language and a processor. The language is based on XTM (Park and Hunting, 2003) and the processor is written in Java, available for running standalone or as a plug-in for Ontopia Omnigator (Ontopia, 2002).

\subsection{Toma language}

Toma (Pinchuk, 2007) is a TMCL language with syntax very similar to SQL. It is based on path expressions that allow access to all elements in the topic map. This language has constructors like SELECT, UPDATE, INSERT and DELETE; this constructors can be used to query and manipulate the topic map.

This language offers the MERGE statement to enable topic maps fusion. Also it offers the EXPORT statement to export the topic map to XTM. Toma provides functions that modify, convert and aggregate data coming from the topic map. So, this language can be used as a topic maps query language (TMQL) and topic maps manipulation language (TMML).

\section{Case study - e-sell corporation}

A list of requirements for the new language was established by the ISO Working Group - the ISO JTC1 SC34 Project for a TMCL (Nishikawa et al., 2004). Part of this document, consists in a section that presents a case study for a language to constraint any topic map. This case study is about an e-commerce application.

For this use case, we created a topic map that stores information about customers, products and orders made by customers.

From that we will formalise the design decisions we have taken and specify its vocabulary and type system (taxonomy). After that we will add application specific rules in AsTMa!, XTche, Toma and OSL, the constraining languages.

\subsection{The e-sell's ontology}

The objective of the ontology is to define sets of vocabularies along with its meaning that will be used within the framework. Rules or constraints also need to be defined to ensure the rigidity of the topic map framework that is used. This ensures that the information contained within the document is valid.
From the product class we derive subclasses which are the categories of products like: beverage, technology and clothing. Some topics of type 'product' like: wine, radio, television, DVD and phone are created. Another topic that needs to be covered as a class is the customer. From this class we derive subclasses which are the different customer categories like person and company.

Figure 1 shows a graph that represents a small part of e-sell's ontology on Vizigator (Gennusa, 2004). This figure presents main topic types (order, product and customer), the other topic types (person, company, technology and beverage) and the topic instances (order 01, radio, Ronnie Alves, ...).

Figure 1 The e-sell corporation's ontology (see online version for colours)

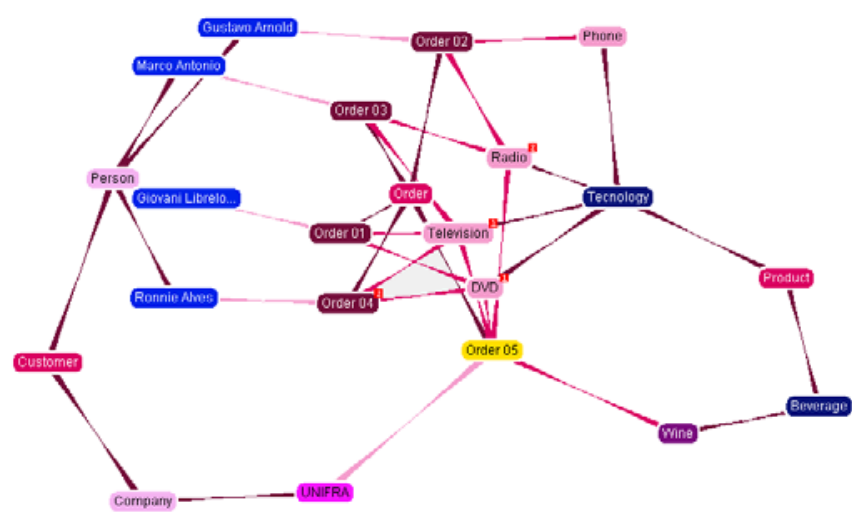

The links in that figure show the relationship between topic type and topic instances (beverage and wine, for instance) or association between two (or more) topics (for instance, order 05 is composed of DVD, radio, television and wine).

\section{Comparing the TMCL}

In this section we will compare the three languages briefly described previously in this paper. Then we point out advantages and disadvantages of each one.

\subsection{Do you need different background to use the languages?}

Yes. To use the XTche language, the topic map designer needs to have solid understanding about XML, XML Schema, XSLT and XPath (Clark and DeRose, 1999). All XTche specifications are in XML Schema format, so the designer can use a visual tool to write the constraints. The constraint can be written in any text editor, but it has the complexity of a common XML Schema.

To specify AsTMa! constraints, the designer is required to know the AsTMa! particular syntactic. To run the AsTMa! processor, it is necessary to have Perl and Prolog compilers installed.

Toma language is SQL-based. It also takes some ideas from Object Orientation notation, Tolog language and AsTMa* syntax. 
OSL language is XTM-based, so the designer needs to specify this kind of constraints in agreement with XTM elements. The OSL tool only requires support for the Java language. Another way to execute OSL verifications is running it on Omnigator (Ontopia, 2002).

\subsection{Do they do the same job?}

Not really. To illustrate this subject, we will present a few comparisons among these languages.

\subsubsection{Validating generic topic map structure}

In the first example, XTche, OSL, Toma and AsTMa! languages virtually do the same job. These three languages allow to verify if a topic map (or a family of topic maps) has some inconsistency in agreement with a set of rules about its structure and content.

For instance, the association is-making-order represents each product line. This creates a relationship between a particular product and an order, along with the quantity of the product ordered. It means that an association of type is-making-order must have three association roles: product, order and quantity. The code below shows the AsTMa! specification:

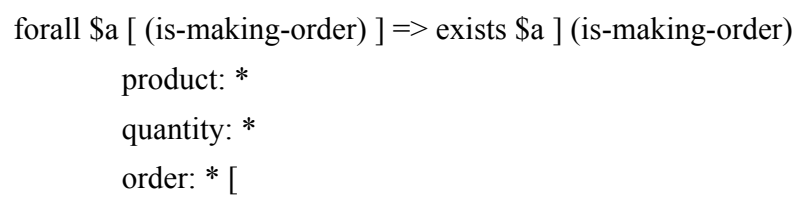

Figure 2 shows a graphical representation of this constraint specified in XTche. The next example presents this XTche constraint in textual format (XML).

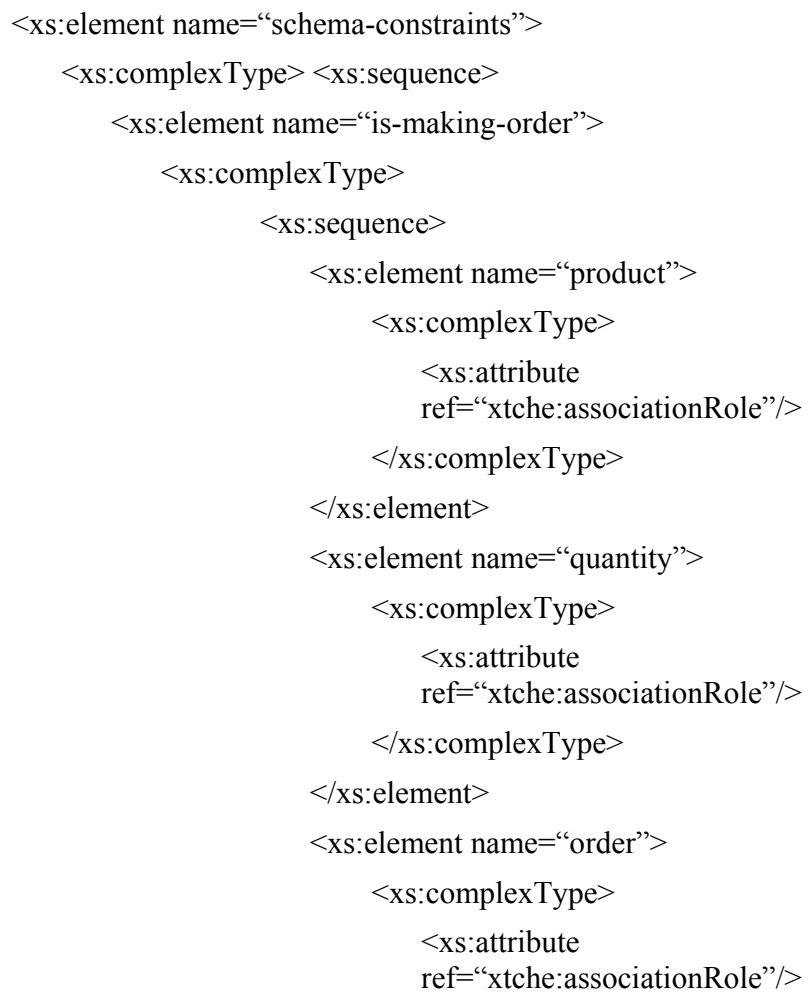

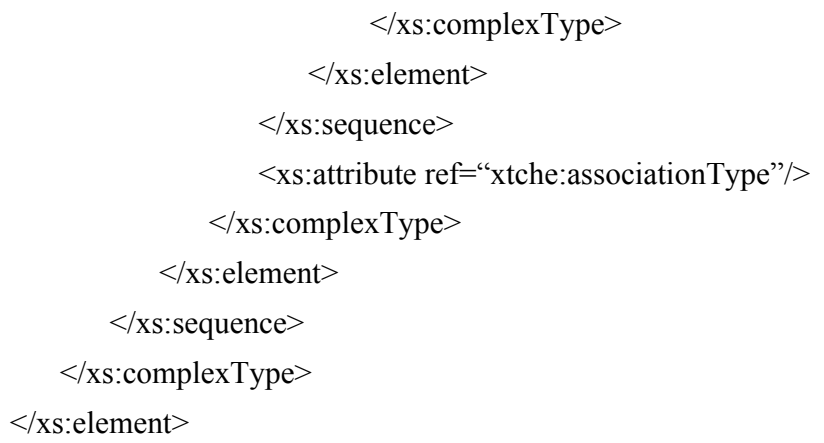

According to the Toma language, the same constraint is specified as shown below:

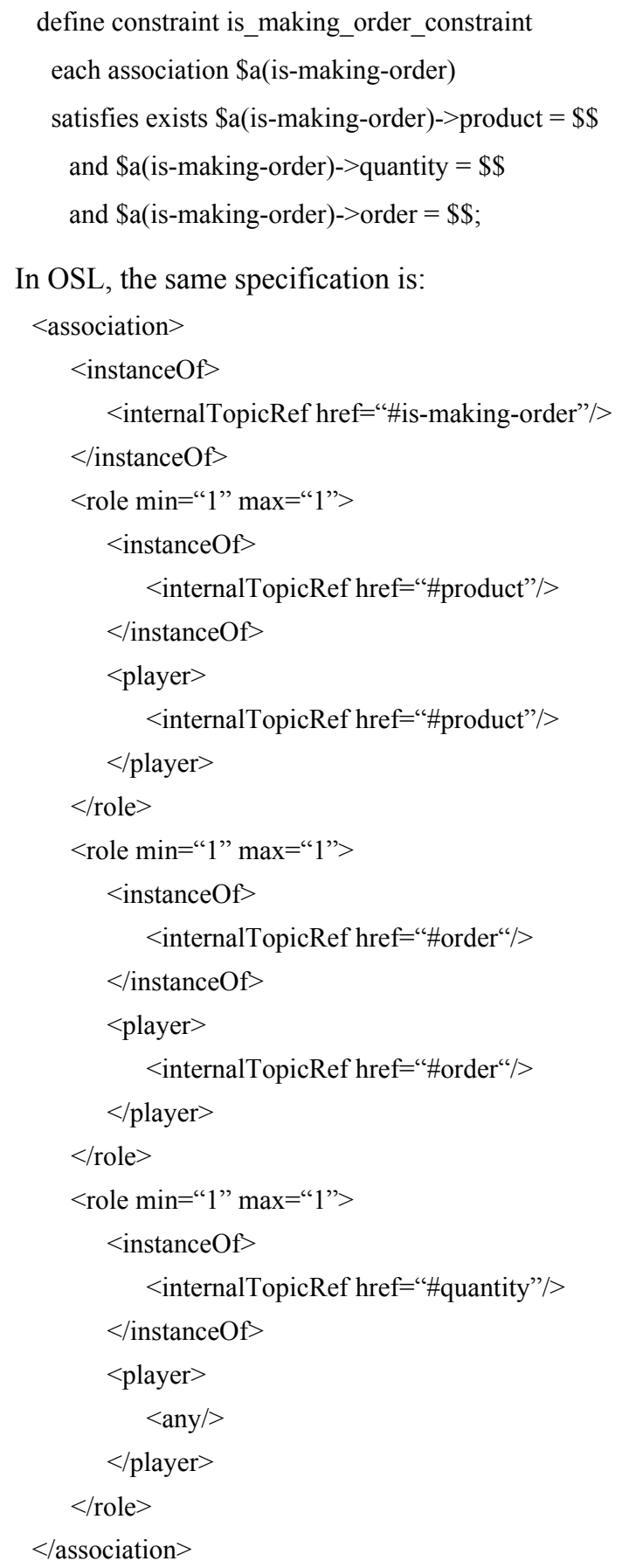


Figure 2 XTche specification for an association structure

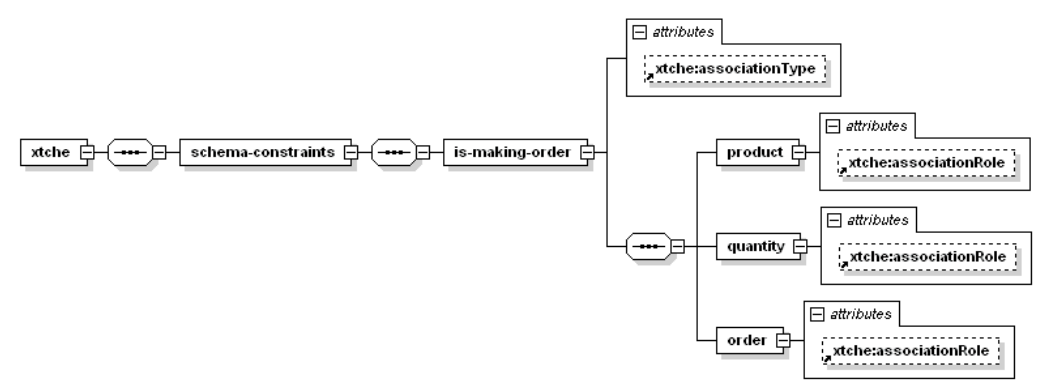

\subsubsection{Validating a specific topic map structure}

In the second example, the constraint is also about the association is-making-order where we need to ensure that a topic instance of product plays the role product and a topic instance of order plays the role order.

The code below introduces the AsTMa! function called exists:

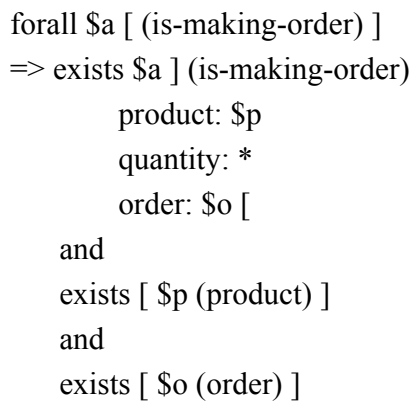

In XTche, the same specification would be:

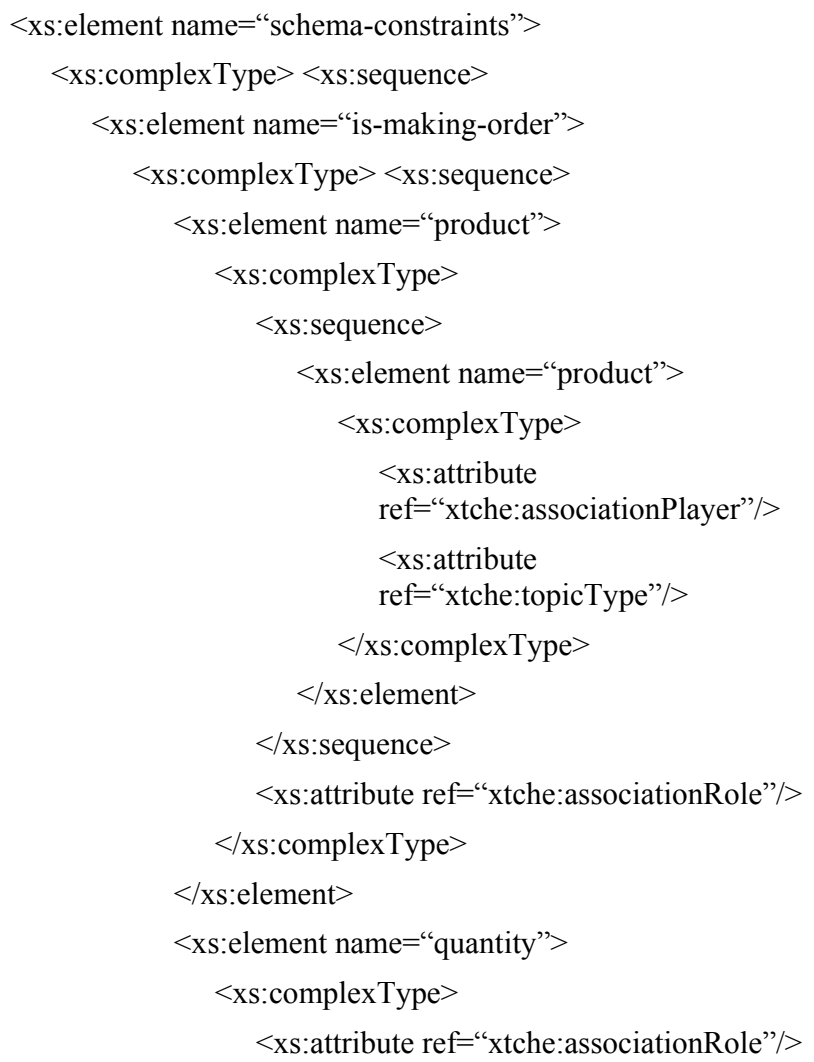

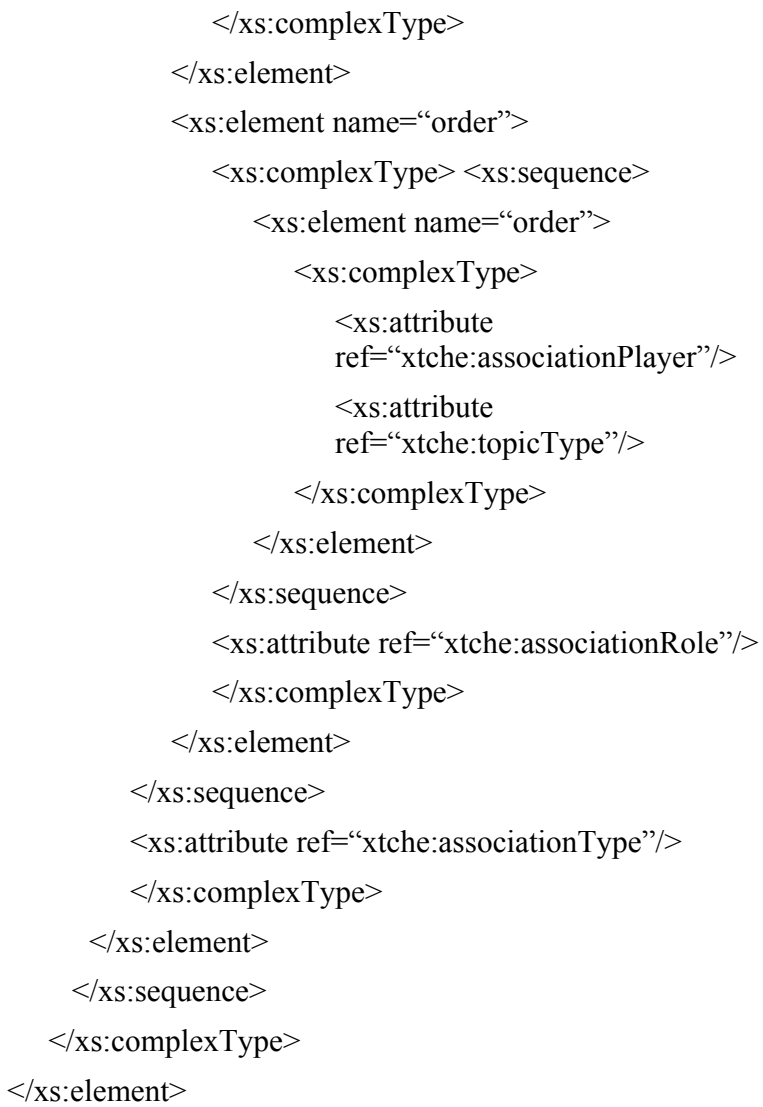

The diagrammatic view of this schema (and the next ones too) can be generated by any XML Schema editor, so we will not show them in the paper.

Toma language defines this constraint like the following:

define constraint is_making_order_constraint_testing_product each association $\$ a$ (is-making-order) satisfies exists $\$ \mathrm{a}$ (is-making-order)->product $=\$ \mathrm{p}$

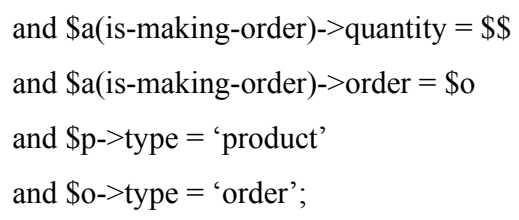

Unfortunately, OSL language does not allow to specify this kind of constraint. 
Figure 3 Data type in XTche language

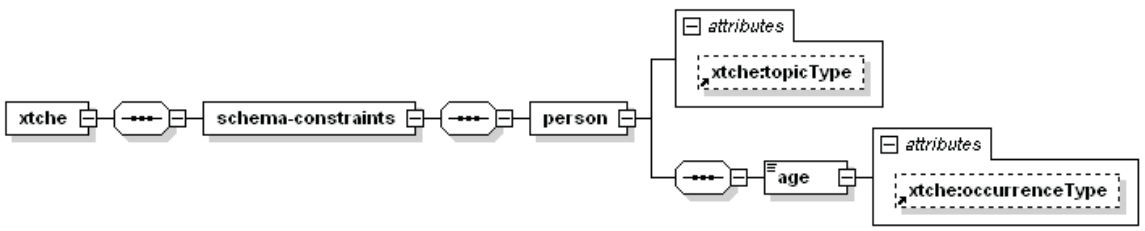

Figure 4 An XTche topic type structure

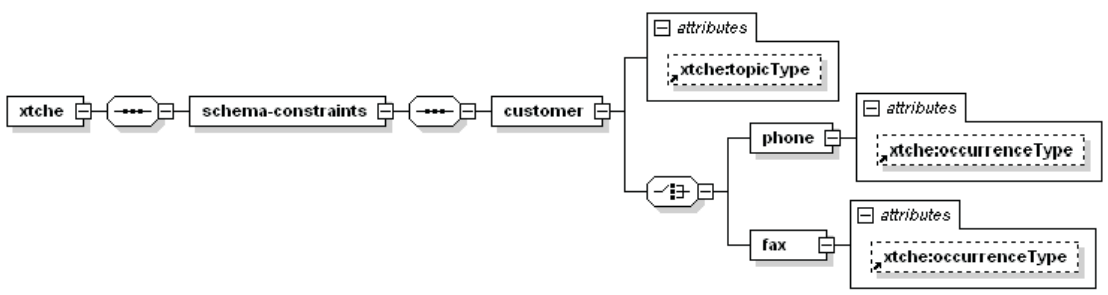

\subsubsection{Data types}

According to the TMDM (Garshol and Moore, 2005), topic maps do have a concept of data and data types, but there is no commitment to any set of primitives such as XML Schema (XSD) (Duckett et al., 2001). That may be a good move, since XSD is - like any other set - quite arbitrary. Useful, but arbitrary. So, if a topic map designer wants to validate an age occurrence as a number, he needs to use a constraint language.

The only way to constrain text in AsTMa! is to use regular expressions. For instance, to allow the invocation of 'boolean test functions', such as:

in (age): ?is_age()

The AsTMa! validator would call this function (implemented externally). According to its creator, this issue would have to be addressed if AsTMa! evolves into a new version.

XTche specification below tests if a person type topic has an age occurrence of integer type (any XSD data type can be used in a XTche specification).

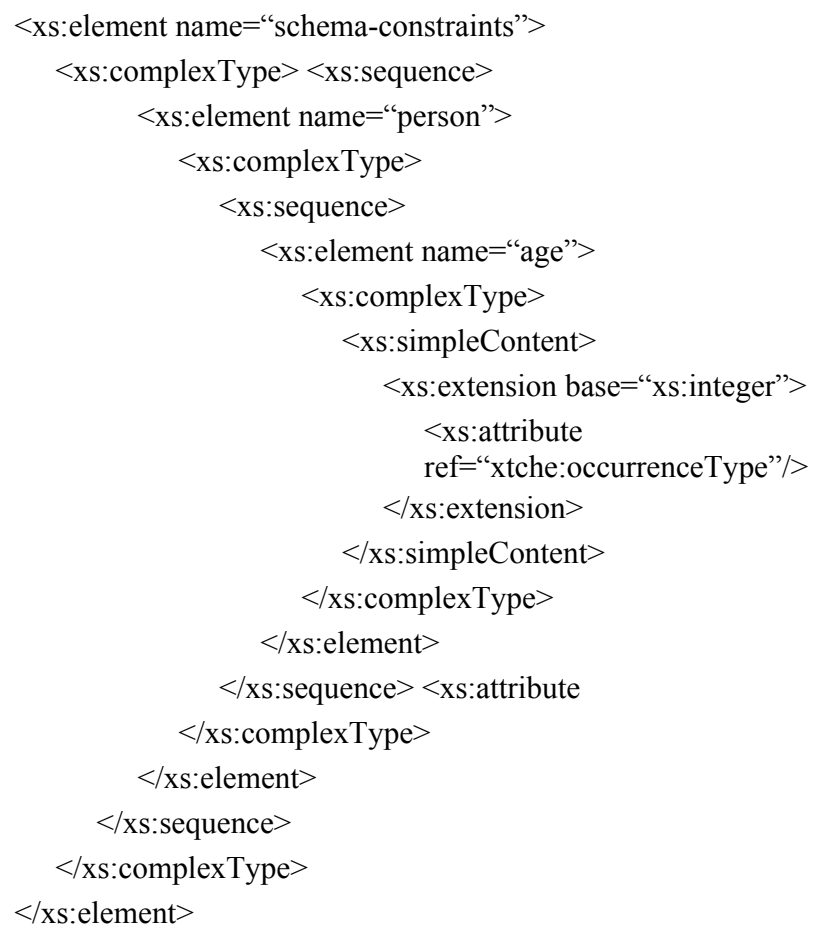

In Figure 3, it is not possible to see the data type specification. But the age element has three horizontal lines that mean this element has a type. In this case, $<$ xs:extension base="xs:integer" $>$ is the type of that element.

Toma has functions to convert parameters. The function to num converts text to a number. The function to_unit converts between units defined by Units Conversion Library by Maio Fundation (http://sourceforge.net/docman/display_doc.php?docid=281 0\&group_id=19449\#SECTd0e169). However, this language does not allow the user to use all XSD data types as XTche does.

In terms of data type, OSL does not provide any kind of data type.

\subsection{Where each language shows its strength}

The topic maps constraints about topics and association structures are easier to specify in these four languages. For instance, the constraint 'customer must have a contact number which is either a phone or a fax number' is specified in AsTMa! like this:

$$
\begin{aligned}
& \text { forall } \$ \mathrm{c}[* \text { (customer) }] \\
& \Rightarrow>\text { exists } \$ \mathrm{c}[\text { in (phone): * ] } \\
& \quad \text { or } \\
& \quad \text { exists } \$ \mathrm{c}[\text { in (fax): * ] }
\end{aligned}
$$

According to the XTche language, the respective specification is shown below and a diagrammatic view of this specification is shown in Figure 4.

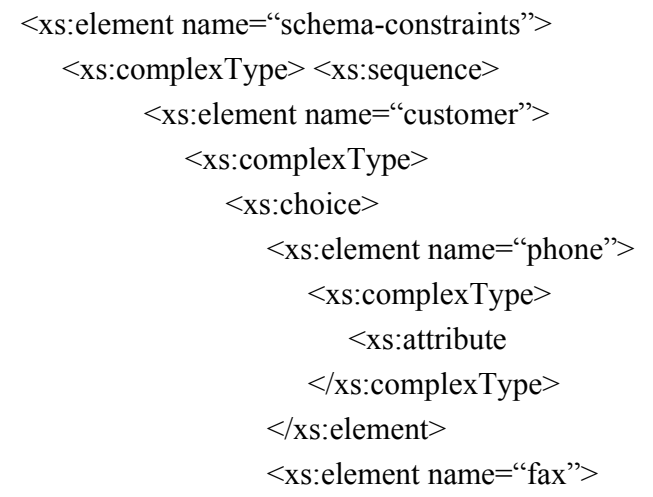




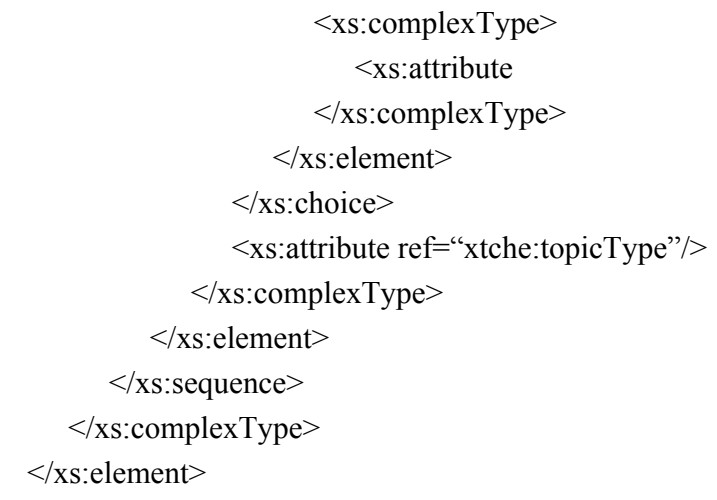

The code below shows the Toma specification for this kind of constraint:

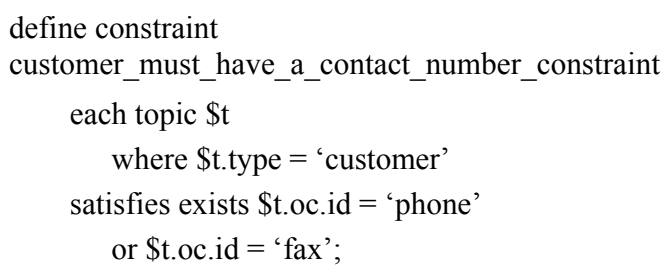

In other hand, OSL correspondent code is presented below. However, this language has a limitation: it does not work with boolean operations. So the constraint 'either a phone or a fax number' is not supported.

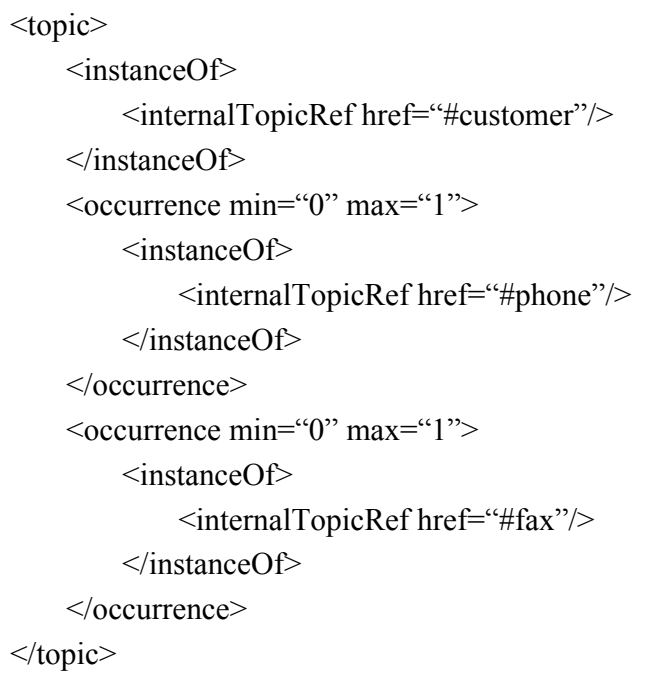

The code above defines a topic instance of customer that has zero or one phone occurrence and zero or one fax occurrence. But, according to this OSL specification, there is no way to verify if a topic instance of customer has both occurrences.

\subsection{Is it possible to use them in similar situations (the same topic maps instances)?}

It is possible to use them in several similar situations but it is important to care about the topic map format. XTche language only processes topic maps in XTM format. There is a small project in the XTche context to create a processor that converts other topic maps formats - linear topic map (LTM) (Garshol, 2002) and HyTime topic maps (HyTM) (Newcomb et al., 2003) - to XTM.
In the same perspective, AsTMa! language only processes topic maps that are in $\mathrm{AsTMa}=$ format.

Toma can not generate TM or XML content. Toma assumes that users that want to create applications using Toma will use in addition other technologies (Java, Perl, Python, etc.). Each of those technologies provides sets of techniques and methodologies to create XML content as well as any other content.

Talking about OSL, this language is part of Omnigator tool (Ontopia, 2002). Many topic maps formats can be validated according to a set of OSL rules. Ontopia enables the navigation over the following topic map formats: XTM, LTM and HyTM; ontologies in resource description framework (RDF) (Lassila and Swick, 1999) format can be navigated by Omnigator too.

\subsection{May we use them to produce an equal result?}

Maybe. The answer is Yes if the topic map designer wants to validate the topic map schema because these three languages confirm the validity of a topic map instance across a set of rules. The answer is No if the topic maps designer wants to validate the topic map with particular constraints, like existence, boolean and conditional constraints. In this case, XTche, Toma and AsTMa! have constructors to specify that; OSL has not.

For example, the constraint 'for all topic that has the topic type customer, it must have a basename (for customer name), an occurrence (for address), a subject identifier (for customer id) and optionally additional occurrence (for e-mail address)' (Nishikawa et al., 2004) can be constrained in XTche, AsTMa!, Toma and OSL. The result for all these languages is a list of the topics that are not conformed with this rule. If all the topics conforms this rule, the result is the topic map validation confirmed. So, for this case: yes, we may use them to produce an equal result.

However another constraint example: 'for all association of is-making-order type, it must have the association roles customer and order played by the topic that is of type customer and order respectively' (Nishikawa et al., 2004) can be validated by Toma, XTche and AsTMa! languages and cannot be validated by OSL language. Thus, for this case: no, we may not use them to produce an equal result.

\subsection{How do AsTMa!, OSL, Toma and XTche relate to TMCL?}

Toma, XTche and AsTMa! languages are based on a draft version of the TMCL, so they are able to specify almost any kind of constraint suggested by TMCL requirement. Toma and AsTMa! do not have constructors to constrain data types.

Toma, AsTMA! and XTche have constructors to make complex conditional, boolean and existential constraints. On the other hand, OSL does not have relationship with TMCL, and it was defined to make just simple validations in a topic map. So the language does not have boolean, existential and conditional operators, becoming a real alternative only in simple and small projects. 
OSL was not designed on the basis of TMCL requirements; it is intended only for validating the topic maps structures. For instance, OSL cannot specify the following constraint: 'topic radio cannot be used to scope association'.

\section{Conclusions}

This paper showed a comparison among the three TMCL-based languages - AsTMa!, OSL, Toma and XTche - over several kinds of topic maps constraints in many different instances. We started with our strong motivation to check a topic map for syntactic and semantic correctness as a notation to describe an ontology that supports a sophisticated computer system (like the applications in the area of Semantic Web or archiving) its validation is crucial!

In order to compare these languages, we succeeded in applying a case study - e-commerce application [subsection 6.1 of TMCL requirements (Nishikawa et al., 2004)] - virtually representative of all possible cases. This means that: on one hand, we were able to describe the constraints required by each problem in a direct, clear and simple way; on the other hand, the topic maps semantic validator could process every document successfully, that is, keeping silent when the constraints are satisfied and detecting/reporting errors whenever the conditions are broken.

Doing a comparison among these languages, some advantages of XTche emerge:

1 XTche has a XML Schema-based language, a well-known format

2 XTche allows the use of an XML Schema graphic editor, like XMLSpy. In a diagrammatic view, it is easy to check visually the correctness of the specification

3 XTche gathers in one specification both the structure and the semantic descriptions and it realises a fully declarative approach requiring no procedural knowledge for users.

The main problem about XTche is the size of this code. If a topic map designer does not have a XML Schema editor, the specification is too complex in a comparison with other languages. This XTche problem is a Toma and AsTMa! advantage: the size of AsTMa! constraints are small, very similar to regular expressions and SQL, respectively.

In a related work, Freese (2002) says that it should be possible to use the DAML+OIL language to provide a constraint and validation mechanism for topic map information. The cited paper discusses how to describe validation and consistency of the information contained in topic maps using DAML+OIL and RDF, showing how to extend XTM and how to define PSIs and class hierarchies, as well as to assign properties to topics.

The main conclusion is that XTche, Toma and AsTMa! comply with almost all requirements stated for TMCL whereas OSL just includes topic maps structure validation.

\section{References}

Barta, R. (2003) AsTMa!, Bond University, TR, available at http://astma.it.bond.edu.au/constraining.xsp.

Biezunsky, M., Bryan, M. and Newcomb, S. (1999) ISO/IEC 13250 - Topic Maps, ISO/IEC JTC 1/SC34, available at http://www.y12.doe.gov/sgml/sc34/document/0129.pdf.

Clark, J. and DeRose, S. (1999) XML Path Language (XPath) - Version 1.0, November, available at http://www.w3.org/TR/xpath.

Dodds, L. (2001) 'Schematron: validating XML using XSLT', XSLT UK Conference, Keble College, Oxford, England.

Duckett, J., Griffin, O., Mohr, S., Norton, F., Ozu, N., Stokes-Rees, I., Tennison, J., Williams, K. and Cagle, K. (2001) Professional XML Schemas, Wrox Press.

Freese E. (2002) 'Using DAML+OIL as a constraint language for topic maps', in $X M L$ Conference and Exposition 2002, IDEAlliance, available at http://www.idealliance.org/papers/xml02/dx_xml02/papers/05 -03-03/05-03-03.html.

Garshol, L.M. (2002) LTM - The Linear Topic Map Notation, Ontopia, available http://www.ontopia.net/topicmaps/ltm.html.

Garshol, L.M. (2004) The Ontopia Schema Language - Reference Specification, available http://www.ontopia.net/omnigator/docs/schema/spec.html.

Garshol, L.M. and Moore, G. (2005) Topic Maps - Data Model, in ISO/IEC JTC 1/SC34, January, available at http://www.isotopicmaps.org/sam/sam-model/.

Gennusa, P. (2004) 'Ontopia's Vizigator(tm) - now you see it!', in $X M L 2004$ Conference and Exposition, IDEAlliance, Washington DC, USA.

Jacinto, M.H., Librelotto, G.R., Ramalho, J.C. and Henriques, P.R. (2003) 'XCSL: XML constraint specification language', CLEI Electronic Journal.

Lassila, O. and Swick, R.R. (1999) 'Resource description framework (RDF) model and syntax specification', World Wide Web Consortium, February, available at http://www.w3.org/TR/REC-rdf-syntax.

Librelotto, G.R., Ramalho, J.C. and Henriques, P.R. (2005) 'Constraining topic maps: a TMCL declarative implementation', in Extreme Markup Languages 2005: Proceedings, IDEAlliance.

Newcomb, S.R., Biezunski, M. and Bryan, M. (2003) The HyTime Topic Maps (HyTM) Syntax 1.0, ISO/IEC JTC 1/SC34 N0391, available http://www.jtc1sc34.org/repository/0391.htm.

Nishikawa, M., Moore, G. and Bogachev, D. (2004) Topic Map Constraint Language (TMCL) Requirements and Use Cases, ISO/IEC JTC 1/SC34 N0548, available at http://www.jtc1sc34.org/repository/0548.htm.

Ontopia (2002) The Ontopia Omnigator, available at http://www.ontopia.net/omnigator/.

Park, J. and Hunting S. (2003) XML Topic Maps: Creating and Using Topic Maps for the Web, ISBN 0-201-74960-2, Addison Wesley.

Pinchuk, R. (2007) TopiWriter User Manual - Toma.

Rath, H.H. (2003) White Paper: The Topic Maps Handbook, Empolis. 